\title{
Transfer map approach to and optical effects of energy degraders in fragment separators
}

\author{
B. Erdelyi* \\ Department of Physics, Northern Illinois University, DeKalb, Illinois 60115, USA \\ and Physics Division, Argonne National Laboratory, Argonne, Illinois 60439, USA \\ L. Bandura \\ Physics Division, Argonne National Laboratory, Argonne, Illinois 60439, USA \\ and Department of Physics, Northern Illinois University, DeKalb, Illinois 60115, USA \\ J. Nolen \\ Physics Division, Argonne National Laboratory, Argonne, Illinois 60439, USA
}

(Received 5 June 2008; published 22 January 2009)

\begin{abstract}
A second order analytical and an arbitrary order numerical procedure is developed for the computation of transfer maps of energy degraders. The incorporation of the wedges into the optics of fragment separators for next-generation exotic beam facilities, their optical effects, and the optimization of their performance is studied in detail. It is shown how to place and shape the degraders in the system such that aberrations are minimized and resolving powers are maximized.
\end{abstract}

DOI: 10.1103/PhysRevSTAB.12.014003

PACS numbers: 41.75. $-\mathrm{i}, 41.85 .-\mathrm{p}, 42.15 . \mathrm{Fr}$

\section{INTRODUCTION}

Exotic nuclei are of interest in a wide variety of contexts ranging from basic nuclear physics to medical applications [1-3]. Several next-generation facilities that use high energy heavy ion driver accelerators for the production of these isotopes in large quantities are presently under commissioning, construction, or envisioned [4-7]. If the production mechanism involves in-flight projectile fragmentation and/or fission of an energetic heavy ion beam, fragment separators are used to capture, separate, and transport the rare-isotope beams to experimental stations [8-13]. Often, the optics of the fragment separators consists of, perhaps several, high order achromatic imaging stages that are necessary for a high purity separation. We studied their optics in a previous publication [14]. It was emphasized that the reaction kinematics induces a large phase space volume of the exotic beam, which requires accurate modeling of the dynamics and correction of aberrations. These requirements can be fulfilled to a large extent by a high order achromat with some additional constraints, as presented in [14]. The optics layout in [14] was based on several symmetries that allowed designing the fragment separator optics with a minimum number of magnets, minimizing overall beam size, vertical beam size in the dipoles, typically all aberrations to third order below $1 \mathrm{~mm}$ in magnitude, reasonably low residual aberrations, and maximal decoupling of aberrations of different orders. However, electromagnetic fields can be made dispersive in mass over charge ratio, but not separately mass and independently charge dispersive. For isotope separation the so-called $B \rho-\Delta E-B \rho$ separation method is

*erdelyi@anl.gov invoked, which requires the utilization of shaped energy degraders [15]. The energy degrader may be considered an optical element, and its influence on the optics can be studied by map methods [16]. In this paper we lay the theoretical basis for the map methods approach to beammaterial interactions and present studies on the optical effects of energy degraders in fragment separators and their mitigation. We restrict ourselves to a one-stage separator since the extension of the results to two-stage separators or one-and-a-half-stage separators for gas cells are straightforward.

The existence of the high order achromatic lattice, based on symmetries, is an essential prerequisite for a high performance fragment separator [14]. The full fragment separator, which includes the energy degraders, in general will not preserve these symmetries. This is mainly due to the energy-loss process taking place in the regions of beam-material interaction. Viewed as an optical element, the map approach can capture the average energy loss of a particle that is dependent on the projectile-target combination, the energy of the reference particle, the shape of the energy degrader, and the initial conditions of the particle. Overall, in suitable coordinates the map of the energy degrader will be the map of a drift with energy having a complex functional dependence on initial conditions. The challenge is then to insert the energy degrader into the optical lattice in such a way to not disturb the symmetries. Unfortunately, this is not possible exactly, but an appropriate location, thickness, and shape can be chosen so that most aberrations remain minimized. This statement will be illustrated below both analytically and numerically.

Once the aberrations are minimized, the quality factors that characterize the performance of the fragment separators are the various resolution and transmission properties. 
We study some of these in detail and draw some conclusions regarding optimality. Of course, to study some of the properties that are important in practice, for example, separation purity, the map approach is not sufficient. The interplay of beam optics and atomic and nuclear processes in the performance must be studied by a hybrid mapMonte-Carlo approach [17-19], and will be the subject of a future publication. However, it is important to note that the effects amenable to the map approach set the upper bound for the performance of the fragment separators [20]. Hence, maximizing this upper bound is the subject of this paper. The inclusion of the nondeterministic effects will deteriorate some of the properties of the fragment separator, but the amount of deterioration is only weakly dependent on the details of the optics.

The organization of the paper is as follows: in Sec. II a second order analytic theory is presented. Section III contains the arbitrary order numerical map computation procedure. Section IV illustrates the optical effects of the wedge by some numerical examples, and presents some studies of resolution of a fragment separator. Finally, we conclude with a brief summary.

\section{SECOND ORDER ANALYTIC THEORY}

In suitable coordinates, the map of the energy degrader is the map of a drift with different energy behavior. For the purpose of the analytical theory we assume that the coordinates used are $\vec{z}=(x, a, y, b, l, \delta)$, where $x, y$ are the horizontal and vertical positions of a particle in the beam, $a, b$ are the ratios of transverse to longitudinal momenta, $l$ is related to the path length, and $\delta$ is the relative momentum deviation with respect to the reference particle. In these geometrical coordinates the transfer map of a magnetic system is invariant with respect to scaling with rigidity; that is, if the magnetic fields are scaled by the ratio of two rigidities the corresponding maps with respect to the two reference particles having those rigidities are identical. The map has six components, the first four being the components of a drift, while the sixth (momentum) component can be written generically to second order as

$$
\begin{aligned}
\delta_{f}= & (\delta \mid x)_{w} x_{i}+(\delta \mid a)_{w} a_{i}+(\delta \mid \delta)_{w} \delta_{i} \\
& +(\delta \mid x x)_{w} x_{i}^{2}+(\delta \mid x a)_{w} x_{i} a_{i}+(\delta \mid a a)_{w} a_{i}^{2} \\
& +(\delta \mid x \delta)_{w} x_{i} \delta_{i}+(\delta \mid a \delta)_{w} a_{i} \delta_{i}+(\delta \mid b b)_{w} b_{i}^{2} \\
& +(\delta \mid \delta \delta)_{w} \delta_{i}^{2},
\end{aligned}
$$

where $\vec{z}_{i}$ are the initial and $\vec{z}_{f}$ are the final coordinates. Throughout the paper map elements with subscript $w$ denote map elements of the wedge.

We are interested to see if the symmetries used in developing the optics solution for a fragment separator without wedges in [14] are maintained or broken by the introduction of the energy degraders in the system. We refer to the details of the definitions and descriptions of these symmetries to [14]. Clearly, the wedge maintains midplane symmetry, but, in general, will break the timeindependence, mirror, and symplectic symmetries used in designing the optics [14]. The question is whether it is possible in special circumstances to regain some of the properties of the mirror-symmetric symplectic system. Because of energy loss, symplecticity is definitely lost because the system fails to be Hamiltonian under these circumstances. Fortunately, as shown in [14], symplecticity enters explicitly in the optical design only at third order. Up to second order, mirror symmetry alone determines the layout. Then, the question is if it is possible to keep the full system, including energy degraders, mirror symmetric. This requires time reversal invariance; that is, if a particle enters the degrader with $\delta_{i}$ and exits with $\delta_{f}$, then backwards in time it would enter with $\delta_{f}$ and exit with $\delta_{i}$ along the same trajectory. This is not impossible a priori since its realization is not forbidden by any fundamental physics law of energy loss of heavy charged particles in materials.

Because of beam-material interactions in energy degraders, the multiple Coulomb scattering induces some detrimental stochastic effects. Therefore, the amount of material in the beam line should be minimized to the extent possible. As a consequence, ideally only one degrader should be utilized per stage. According to the paragraph above, it should be placed at a mirror-symmetric point of the fragment separator. It follows that if a particle enters the mirror-symmetrically placed degrader with $\delta_{i}$ then it should exit the degrader with the same $\delta_{i}$. In other words, the energy degrader should preserve the existing dispersion without the degrader. Looking at Eq. (1), it follows that this requires two things: $(\delta \mid x)_{w} \neq 0$ and $x_{i}$ should be $\delta_{i}$ dependent, that is the degrader should be placed in a dispersive region of the beam line. $(\delta \mid x)_{w}$ being nonzero implies that the degrader should have a thickness variation in the $x$ direction. Hence, the degrader must be wedge shaped. This will also induce a nonzero $(\delta \mid a)_{w}$. To second order, by introducing an edge curvature, we can control $(\delta \mid x x)_{w}$, and by adjusting the thickness $(\delta \mid \delta)_{w}$ or $(\delta \mid \delta \delta)_{w}$ to some extent.

In order to see explicitly the aberrations of the full system, the wedge map needs to be composed with the rest of the lattice. The rest of the mirror-symmetric lattice map, which is a second order achromat shown schematically in Fig. 1, was computed to second order in [14]. The map $\mathcal{M}$ up to the mirror-symmetric point (half the system) is

$$
\begin{gathered}
x_{f}=-x_{i}+(x \mid \delta) \delta_{i}+(x \mid x x) x_{i}^{2}+(x \mid a a) a_{i}^{2}+(x \mid x \delta) x_{i} \delta_{i} \\
+(x \mid y y) y_{i}^{2}+(x \mid b b) b_{i}^{2}+(x \mid \delta \delta) \delta_{i}^{2}, \\
a_{f}=-a_{i}+(a \mid x a) x_{i} a_{i}+(a \mid a \delta) a_{i} \delta_{i}+(a \mid y b) y_{i} b_{i}, \\
y_{f}=-y_{i}+(y \mid x y) x_{i} y_{i}+(y \mid a b) a_{i} b_{i}+(y \mid y \delta) y_{i} \delta_{i}, \\
b_{f}=-b_{i}+(b \mid x b) x_{i} b_{i}+(b \mid a y) a_{i} y_{i}+(b \mid b \delta) b_{i} \delta_{i},
\end{gathered}
$$




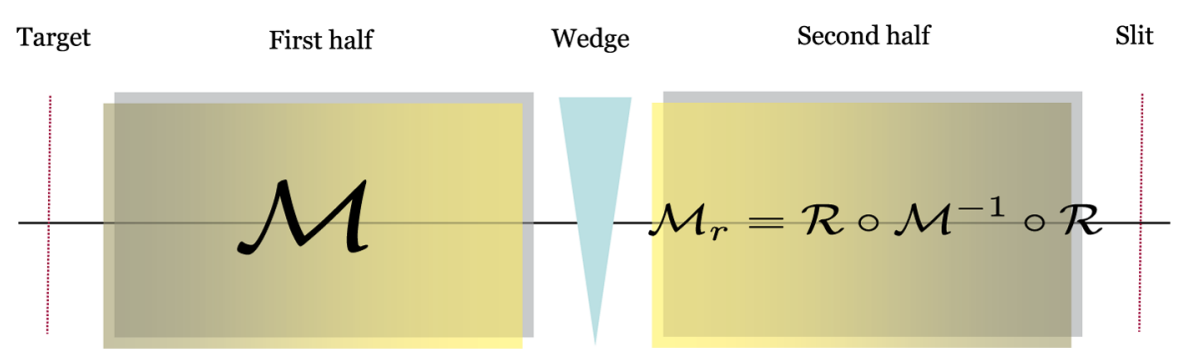

FIG. 1. (Color) Layout of a one-stage separator. The map of the second half is given by symmetry operations. The full system is designed to be a high order achromat when there is no wedge in the middle. The placement of the achromatic wedge at the mirrorsymmetric point of the system should disturb minimally the properties of the initial system.

$$
\delta_{f}=\delta_{i}
$$

followed by the reversed system. The map of the reversed system can be computed by the well-known formula $\mathcal{R} \circ$ $\mathcal{M}^{-1} \circ \mathcal{R}$, where $\mathcal{M}$ is the map of the forward system, and $\mathcal{R}$ is a universal linear map that inverts the signs of $a$ and $b$. The map of the full system up to the achromatic point is identity up to second order in the absence of the wedge, regardless of the specific values of the aberrations present in (3)-(7). For details, see [14]. Since the middle of the system is dispersive and it is a mirror-symmetric point, it satisfies all requirements set forth in the preceding paragraphs for the location of the wedge. If we denote the map represented by (3)-(7) by $\mathcal{M}$, the map of a drift of length $l$ by $\mathcal{M}_{d}(l)$, and the map of the wedge by $\mathcal{M}_{w}$, the total map of the system, including the wedge is

$$
\begin{aligned}
\mathcal{M}_{\text {tot }}= & \mathcal{R} \circ \mathcal{M}^{-1} \circ \mathcal{R} \circ \mathcal{M}_{d}\left(-\frac{l_{w}}{2}\right) \circ \mathcal{M}_{w} \\
& \circ \mathcal{M}_{d}\left(-\frac{l_{w}}{2}\right) \circ \mathcal{M}
\end{aligned}
$$

where $l_{w}$ is the length (central thickness) of the wedge. The composition can be performed analytically by MATHEMATICA [21]. To first order, we obtain the following transfer matrix:

$$
M_{\mathrm{tot}}=\left(\begin{array}{ccccc}
1-(x \mid \delta)(\delta \mid x)_{w} & (x \mid \delta)\left[\frac{l_{w}}{2}(\delta \mid x)_{w}-(\delta \mid a)_{w}\right] & 0 & 0 & (x \mid \delta)\left[(x \mid \delta)(\delta \mid x)_{w}+(\delta \mid \delta)_{w}-1\right] \\
0 & 1 & 0 & 0 & 0 \\
0 & 0 & 1 & 0 & 0 \\
0 & 0 & 0 & 1 & 0 \\
-(\delta \mid x)_{w} & \frac{l_{w}}{2}(\delta \mid x)_{w}-(\delta \mid a)_{w} & 0 & 0 & (x \mid \delta)(\delta \mid x)_{w}+(\delta \mid \delta)_{w}
\end{array}\right)
$$

There are two free parameters that we can choose: the length and the opening angle of the wedge. These two parameters can be used to simplify (9). In practice, due to the reaction kinematics and target thickness, the initial angles and energy spreads are large, while the primary beam spots are very small. This entails that we should use the two wedge parameters to minimize $(x \mid a)_{\text {tot }}$ and $(x \mid \delta)_{\text {tot }}$. The angle will determine $(\delta \mid x)_{w}$ while the length $l_{w}$ will influence $(\delta \mid \delta)_{w} .(\delta \mid a)_{w}$ will change too, but we cannot control its value. Therefore, the fit of the angle and length to satisfy simultaneously the following two equations (for a given beam-wedge combination),

$$
\begin{gathered}
\frac{l_{w}}{2}(\delta \mid x)_{w}-(\delta \mid a)_{w}=0, \\
(x \mid \delta)(\delta \mid x)_{w}+(\delta \mid \delta)_{w}-1=0,
\end{gathered}
$$

determines the optimal wedge parameters from the point of view of the optics. Hence, (9) simplifies to

$$
M_{\mathrm{tot}}=\left(\begin{array}{ccccc}
(\delta \mid \delta)_{w} & 0 & 0 & 0 & 0 \\
0 & 1 & 0 & 0 & 0 \\
0 & 0 & 1 & 0 & 0 \\
0 & 0 & 0 & 1 & 0 \\
-(\delta \mid x)_{w} & 0 & 0 & 0 & 1
\end{array}\right)
$$

It is interesting to note that (10) is in fact a geometrical condition that is always satisfied, independent of any detail of the system. Its proof can be found in the Appendix. This fact implies that the wedge maintains the imaging property of the system at any thickness, and for a given thickness one just needs to find the angle that cancels the total dispersion. Therefore, the thickness remains a free parameter that may be used to optimize other quantities such as the resolution. Moreover, this condition serves as a check of the numerical procedure for the computation of the wedge map.

The transfer matrix (12) shows that it is not possible to recover the identity map exactly when the wedge is inserted into a mirror-symmetric achromat. The system will have magnification $(\delta \mid \delta)_{w}>1$ and will have an increased momentum spread (proportional to the initial beam size). 
The determinant being equal to $(\delta \mid \delta)_{w}$ also entails an increase in horizontal emittance. Again, fortunately the initial beam size usually is very small, so the momentum-spread effect is not very serious, but the emittance growth could be considerable, depending on the case.

The second order part of $\mathcal{M}_{\text {tot }}$ is complicated. However, under the realistic constraints of (10) and (11) and $x_{i}, y_{i} \rightarrow$ 0 the results simplify to

$$
\begin{gathered}
\begin{array}{r}
x_{f}={ }_{2}(x \mid a a)_{\mathrm{tot}} a_{i}^{2}+(x \mid a \delta)_{\mathrm{tot}} a_{i} \delta_{i}+(x \mid b b)_{\mathrm{tot}} b_{i}^{2} \\
+(x \mid \delta \delta)_{\mathrm{tot}} \delta_{i}^{2},
\end{array} \\
a_{f}={ }_{2} 0, \\
y_{f}={ }_{2} 0, \\
b_{f}={ }_{2} 0, \\
\delta_{f}={ }_{2}(\delta \mid a a)_{\mathrm{tot}} a_{i}^{2}+(\delta \mid a \delta)_{\mathrm{tot}} a_{i} \delta_{i}+(\delta \mid b b)_{\mathrm{tot}} b_{i}^{2} \\
+(\delta \mid \delta \delta)_{\mathrm{tot}} \delta_{i}^{2},
\end{gathered}
$$

where the $x_{f}$ elements in the total map are

$$
\begin{aligned}
(x \mid a a)_{\mathrm{tot}}= & (x \mid a a)\left[1-(\delta \mid \delta)_{w}\right]-\frac{l_{w}^{2}}{4}(\delta \mid x x)_{w}(x \mid \delta) \\
+ & \frac{l_{w}}{2}(\delta \mid x a)_{w}(x \mid \delta)+(\delta \mid a a)_{w}(x \mid \delta), \\
(x \mid a \delta)_{\mathrm{tot}}= & l_{w}(\delta \mid x x)_{w}(x \mid \delta)^{2}-(\delta \mid x a)_{w}(x \mid \delta)^{2} \\
& +\frac{l_{w}}{2}(\delta \mid x \delta)_{w}(x \mid \delta)-(\delta \mid a \delta)_{w}(x \mid \delta), \\
(x \mid b b)_{\mathrm{tot}}= & (x \mid b b)[1-(\delta \mid \delta)]+(\delta \mid b b)_{w}(x \mid \delta), \\
(x \mid \delta \delta)_{\mathrm{tot}}= & (x \mid \delta \delta)[1-(\delta \mid \delta)]+(\delta \mid x x)_{w}(x \mid \delta)^{3} \\
& +(\delta \mid x \delta)_{w}(x \mid \delta)^{2}+(\delta \mid \delta \delta)_{w}(x \mid \delta),
\end{aligned}
$$

and similarly complicated functions for $(\delta \mid \cdots)_{\text {tot }}$.

Out of the second order wedge map elements we can control $(\delta \mid x x)_{w}$ only, by curving the edges. Equation (13) implies that in principle we can cancel all $x_{f}$ aberrations by judiciously selecting four aberration coefficients we can control, namely $(x \mid a a),(x \mid b b),(x \mid \delta \delta)$ (these three are determined by the pure magnetic optics and may assume arbitrary values according to the achromat theory), and $(\delta \mid x x)_{w}$. Solving the system of Eqs. (18)-(21) we obtain

$$
\begin{gathered}
(x \mid a a)=\frac{1}{(\delta \mid x)_{w}}\left[\frac{l_{w}}{4}(\delta \mid x a)_{w}+\frac{l_{w}^{2}}{8(x \mid \delta)}(\delta \mid x \delta)_{w}\right. \\
\left.-\frac{l_{w}}{4(x \mid \delta)}(\delta \mid a \delta)_{w}-(\delta \mid a a)_{w}\right], \\
(x \mid b b)=-\frac{(\delta \mid b b)_{w}}{(\delta \mid x)_{w}},
\end{gathered}
$$

$$
\begin{gathered}
(x \mid \delta \delta)=\frac{1}{(\delta \mid x)_{w}}\left[\frac{(x \mid \delta)^{2}}{l_{w}}(\delta \mid x a)_{w}-\frac{(x \mid \delta)}{2}(\delta \mid x \delta)_{w}\right. \\
\left.-\frac{(x \mid \delta)}{l_{w}}(\delta \mid a \delta)_{w}-(\delta \mid \delta \delta)_{w}\right] \\
(\delta \mid x x)_{w}=\frac{(\delta \mid x a)_{w}}{l_{w}}-\frac{(\delta \mid x \delta)_{w}}{2(x \mid \delta)}+\frac{(\delta \mid a \delta)_{w}}{l_{w}(x \mid \delta)}
\end{gathered}
$$

Interestingly, if (22)-(25) are satisfied we obtain that all energy aberrations vanish simultaneously, $(\delta \mid a a)_{\text {tot }}=$ $(\delta \mid a \delta)_{\text {tot }}=(\delta \mid b b)_{\text {tot }}=(\delta \mid \delta \delta)_{\text {tot }}=0$. Therefore, in principle the full system including the wedge can be made completely aberration-free up to second order for a pointlike initial primary beam.

Unfortunately, there is no guarantee that the system of equations always admits a unique solution [especially (24) and (25) are difficult to realize in practice], and even if it does, in principle it might be a different solution for each projectile-beam combination. The difficulty in satisfying (25) lies in the dependence of the left-hand side on the right-hand side. Therefore, any change in the curvature will change not only $(\delta \mid x x)_{w}$ but also $(\delta \mid x a)_{w}$, for example. Zeroing out (24) would require additional strong multipoles in the system. In case there is no solution canceling exactly the aberrations numerical optimization can be employed to minimize them. The advantage of the numerical optimization is that it can be easily extended to arbitrary high orders. For the practical tuning of the fragment separator, perhaps the simplest method is to notice that in general $l_{w}$ is small and the range of typical values of most wedge map elements is limited. Accordingly, (22)(25) for typical cases reduce to

$$
\begin{array}{ll}
(x \mid a a) \approx-\mathcal{O}(1), & (x \mid b b) \approx-\mathcal{O}(1), \\
(x \mid \delta \delta) \approx-\mathcal{O}(1), & (\delta \mid x x) \approx-\mathcal{O}(1),
\end{array}
$$

where $\mathcal{O}(1)$ denotes a number of the order of 1 . Therefore, one does not introduce very large aberrations if typically the fragment separator is tuned so $(x \mid a a),(x \mid b b),(x \mid \delta \delta)$, and $(\delta \mid x x)$ are roughly vanishing, but not exactly zero. If some aberration coefficients in (22)-(25) cannot be made small, then (18)-(21) give insight and help in finding an optimum tune for the fragment separator. For example, according to (18)-(21) a good alternative would be to fit $(x \mid a a)$ and $(x \mid b b)$ to small negative values such that $(x \mid a a)_{\text {tot }}$ and $(x \mid b b)_{\text {tot }}$ vanish, and the curvature to cancel the effect of an arbitrary $(x \mid \delta \delta)$. Since $(x \mid a \delta)_{\text {tot }}$ does not involve second order aberrations due to the magnetic optics, typically it assumes small values that may be neglected. In Sec. IV we study the practical realizations based on these solutions.

Obviously, the analytic theory gets very complicated at third and higher orders and a numerical solution is the only one feasible. In the next section we outline the algorithm 
for the arbitrary order wedge map computation in the differential algebraic (DA) framework [16].

\section{NUMERICAL COMPUTATION OF THE ENERGY DEGRADER'S TRANSFER MAP}

The analytic theory of the previous section gives useful insight into the dynamics with wedges, but is formal in the sense that it does not give the actual numerical values of the wedge map elements and is limited to second order. Over the years, in the nuclear physics community a lot of effort went into writing computer codes that are able to accurately compute the energy loss of heavy ions in materials. We use these results, cast them into differential algebraic form, and use map inversion techniques to compute the analog of (7) numerically to arbitrary order. One of these codes that fits well into our algorithm is ATIMA. It uses a spline interpolation for each projectile-degrader material combination to compute the energy loss and straggling [22]. Although it has huge data files, it is fast and accurate and the piecewise polynomial interpolation makes the evaluation of the energy-loss functions easy to implement in DA. In fact, we implemented the DA version of a function that computes the range of a particle of initial energy $E_{i}$ in some material. If one is interested in the final energy of the particle, after going through a material of thickness $T$ less than the range, the following implicit equation for the final energy $E_{f}$ needs to be solved:

$$
\text { range }\left(E_{i}\right)-\operatorname{range}\left(E_{f}\right)-T=0 \text {, }
$$

where range $(E)$ is given by ATIMA.

To set up the DA version of (27) we need two things: the DA evaluation of the range function and a method to evaluate the effective thickness seen by a particle. The thickness will depend on the length and shape of the wedge, and the initial conditions of the particle. By regarding the entrance and exit edge of the degrader as curved surfaces described by two polynomials, DA allows the projection of the trajectory of an arbitrary particle onto these surfaces and calculation of the distance between the points, giving the required thickness. All along, the explicit dependence on the particle's initial condition is retained. For more details we refer the reader to [23]. Therefore, the DA version of (27) can be written as

$$
\text { range }\left[E_{i, 0}\left(1+\delta_{i}\right)\right]-\operatorname{range}\left[E_{f, 0}\left(1+\delta_{f}\right)\right]-T\left(\vec{z}_{i}\right)=0 \text {, }
$$

where $E_{i, 0}$ and $E_{f, 0}$ are the initial and final energies of the reference particle (here $\delta$ is the relative energy deviation). In general, (28) is a complicated nonlinear multivariable function of the form $f\left(\vec{z}_{i}, \delta_{f}\right)=0$ that needs to be solved for $\delta_{f}$. Assume that $\vec{z}_{i}$ is $n$ dimensional (it may contain parameters such as mass and charge), and introduce the $n$-dimensional identity operator $I_{n}$. Combining $I_{n}$ and $f$ we obtain a $n+1$-dimensional operator that can be re- garded as a map, $\mathcal{N}_{n+1}=\left(I_{n}, f\right)$, and the following equation is obtained:

$$
\mathcal{N}_{n+1}\left(\vec{z}_{i}, \delta_{f}\right)=\left(\vec{z}_{i}, 0\right) .
$$

By construction $\mathcal{N}_{n+1}$ is origin preserving and has a nonvanishing Jacobian determinant at the origin, hence DA methods can be employed for the explicit inversion to arbitrary order [16], and obtain

$$
\left(\vec{z}_{i}, \delta_{f}\right)=\mathcal{N}_{n+1}^{-1}\left(\vec{z}_{i}, 0\right),
$$

from which we can read off the energy component of the wedge map

$$
\delta_{f}=\left[\mathcal{N}_{n+1}^{-1}\left(\vec{z}_{i}, 0\right)\right]_{(n+1) \text { th component }}
$$

Since the other components of the wedge map are the same as those of a drift length equal to the wedge thickness seen by the reference particle, we have the algorithm for the full wedge map. The algorithm was implemented in the code COSY INFINITY [24].

We mention that if time of flight is of interest a similar procedure for the DA-based spline evaluation of the timeof-flight splines could be implemented. Also, if the equations of the motion of a particle in material are available, a DA integration allows the computation of the wedge map from first principles.

\section{NUMERICAL RESULTS AND APPLICATIONS TO FRAGMENT SEPARATORS}

In this section we provide a few numerical examples of wedge maps, optical effects, aberrations induced, and computation of optimal wedge parameters followed by some studies of resolution in a proposed fragment separator for a next-generation exotic beam facility.

\section{A. Energy degrader maps}

The interesting component of the energy degrader map is the energy component, which tells us how the energy of a particle changes relative to a reference particle. As we expect, this quantity will depend on the projectile-target combination, the degrader thickness and shape, and particle initial phase space coordinates. To illustrate the main features of this dependence, Table I summarizes the case of ${ }^{132} \mathrm{Sn}$ particles incident on an aluminum wedge of $30 \%$ of the range thickness at $200 \mathrm{MeV} / \mathrm{u}$ initial energy. The angle and curvature is interpreted in the following way: if the entrance shape of the degrader is regarded as a planar curve $s(x)=c_{1} x+c_{2} x^{2}$, then the angle of the degrader is $2 \arctan \left(c_{1}\right)$ and the curvature is $2 c_{2}$. The exit shape is assumed to be described by the same coefficients. The numbers $c_{1}$ and $c_{2}$ are called the shape coefficients in all subsequent tables and plots. Also, unless otherwise noted, the wedge materials are always aluminum.

Because of symmetry, for a homogeneous block absorber there are no $\left(\delta \mid x^{n} y^{m}\right)$ terms since particles entering 
TABLE I. Some wedge map elements that illustrate the effect of the shape on the map. The numerical values correspond to a $200 \mathrm{MeV} / \mathrm{u}^{132} \mathrm{Sn}$ beam incident on an aluminum degrader of thickness equal to $30 \%$ of the tin beam range.

\begin{tabular}{lcccccc}
\hline \hline Case/map element & $(\delta \mid x)$ & $(\delta \mid a)$ & $(\delta \mid \delta)$ & $(\delta \mid x x)$ & $(\delta \mid a a)$ & $(\delta \mid \delta \delta)$ \\
\hline Zero angle and curvature & 0 & 0 & 1.430081 & 0 & -0.135956 & -0.196226 \\
$10 \mathrm{mrad}$ angle, no curvature & -1.298587 & -0.001359 & 1.430081 & -0.498455 & -0.135963 & -0.196226 \\
$10 \mathrm{mrad}$ angle and $10^{-3}$ curvature & -1.29858 & -0.001359 & 1.430081 & -1.797032 & -0.135966 & -0.196226 \\
\hline \hline
\end{tabular}

the degrader at different horizontal and/or vertical positions will see the same thickness. Particles entering with a nonzero horizontal or vertical angle will see the same thickness regardless of the sign of the angle, implying that only those $\left(\delta \mid a^{n} b^{m}\right)$ terms appear in the map for which $n$ and $m$ are even. Nonzero $\left(\delta \mid \delta^{n}\right)$ terms always appear due to the fact that energy loss is not a linear function of energy.

A nonzero angle will induce a position dependent energy gradient, $(\delta \mid x) \neq 0$, and will break the angular symmetry,

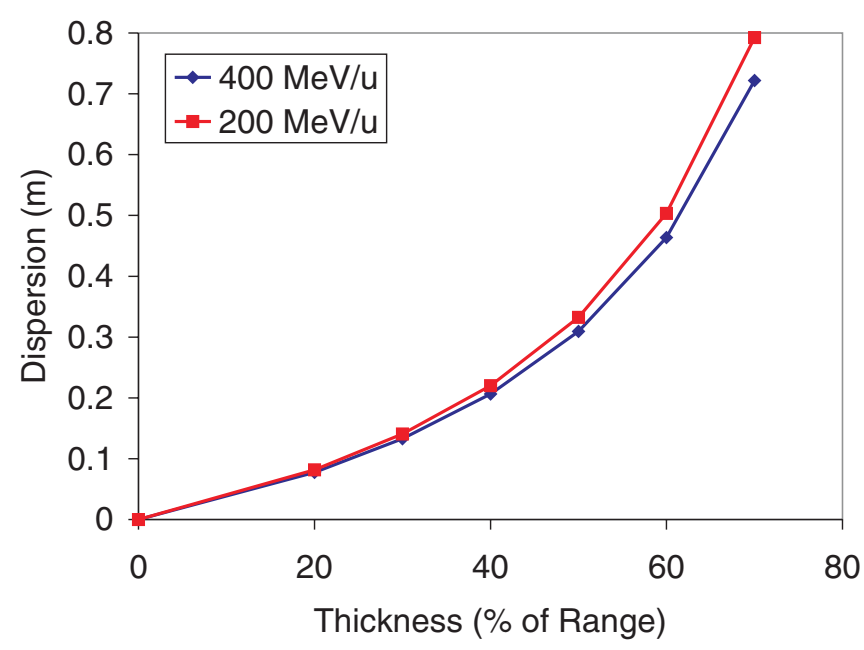

FIG. 2. (Color) Effect of not shaping the degrader: dispersion introduced at the location of the no-wedge achromatic image. $(\delta \mid a) \neq 0$. These two quantities are linked by (10). The main effect of a nonzero curvature can be seen on $(\delta \mid x x)$ as expected. Also, $(\delta \mid a a)$ is practically constant, independent of shape.

\section{B. Optics with absorbers}

Once we know how to compute numerically the maps of absorbers to arbitrary order, we can include them in the layout of fragment separators according to the procedure outlined in the previous sections and calculate the map of the whole system, including absorbers. For all plots below, the following initial phase space is used: $1 \mathrm{~mm}$ spot sizes horizontally and vertically, $\pm 50 \mathrm{mrad}$ divergence in both planes, and $\pm 9 \%$ momentum spread.

First, the dramatic effects the wedges can have on the optics if not shaped correctly are shown in Figs. 2 and 3. The main linear effect of a zero angle is reflected in the induced dispersion and, hence, the image width at the location of the achromatic image in the absence of the wedge (Fig. 2). Also, some of the aberrations become very large (Fig. 3). Notice that all these effects are very weakly dependent on the initial beam energies. Also, it is important to note that all aberrations that become very large are connected to large initial angles of particles. As we will see, these are the main detrimental effects of the wedges that cannot be removed completely even if the optics is perfect and the wedges are shaped ideally.

Next, we fit the wedge angle to satisfy (11) and studied the angle's dependence on a variety of factors. Figure 4
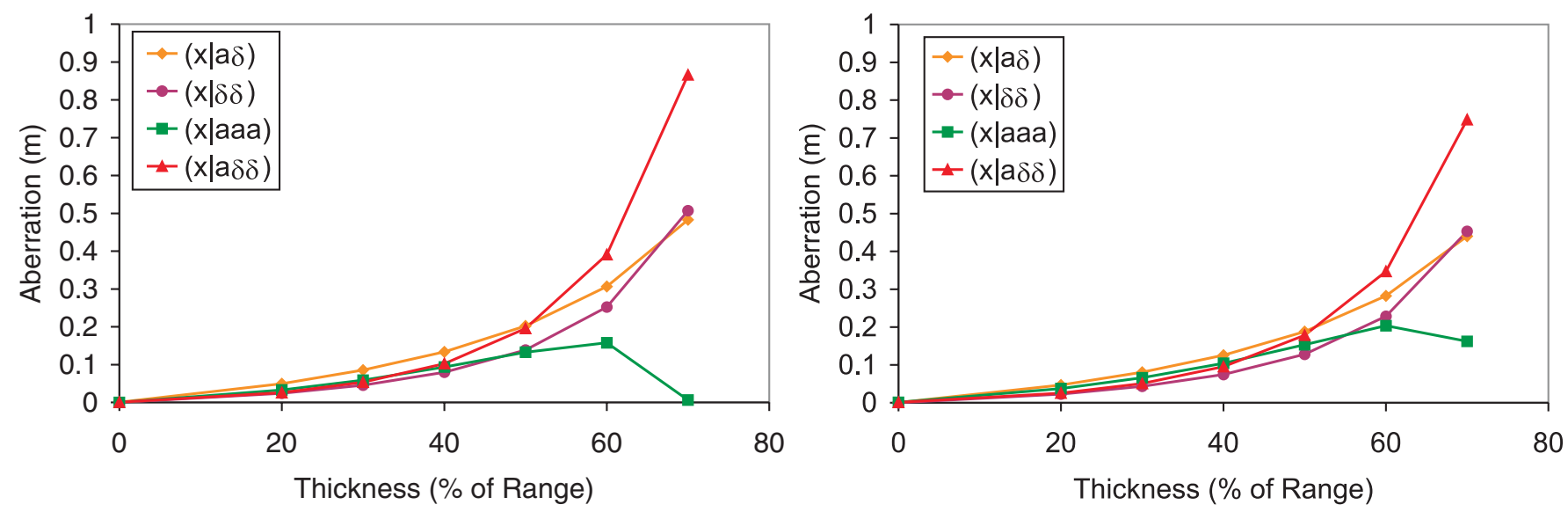

FIG. 3. (Color) Effects of not shaping the degrader: large second and third order aberrations are generated at the no-wedge achromatic image at 200 (left) and 400 (right) $\mathrm{MeV} / \mathrm{u}$ energies. 


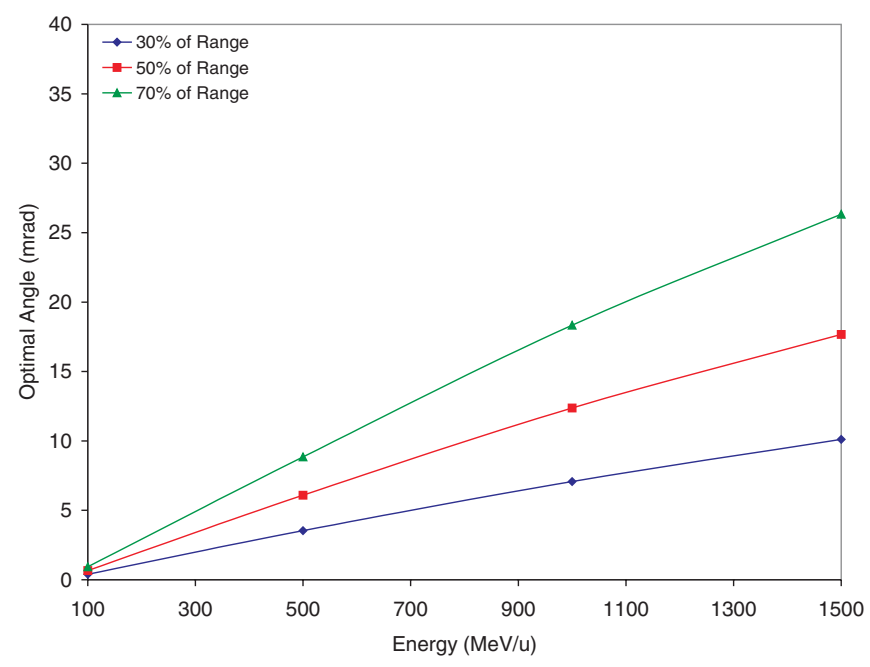

FIG. 4. (Color) Optimal angle needed to maintain linear achromaticity for the case of a ${ }^{100} \mathrm{Sn}$ beam at various energies and three different wedge thicknesses.

shows how the ideal wedge angle varies as a function of incident energy of a ${ }^{100} \mathrm{Sn}$ beam for three different wedge thicknesses. We notice that the needed angle increases with both energy and thickness. The same calculation for heavier particles shows that the optimal angles increase with mass too. Another way of presenting this information is to plot the optimal angle as a function of thickness for fixed initial energies. For ${ }^{132} \mathrm{Sn}$, Fig. 5 shows that at higher energies larger angles are needed at any thickness and the slope of the lines at different energies is different. It is clear that at lower energies the angles needed become very small, making the manufacturing of energy degraders more difficult. The material of the energy degrader also influences the value of the wedge angle. Figure 6 presents the case of two kinds of wedges and many different incident beams. It is assumed that the beams have the same

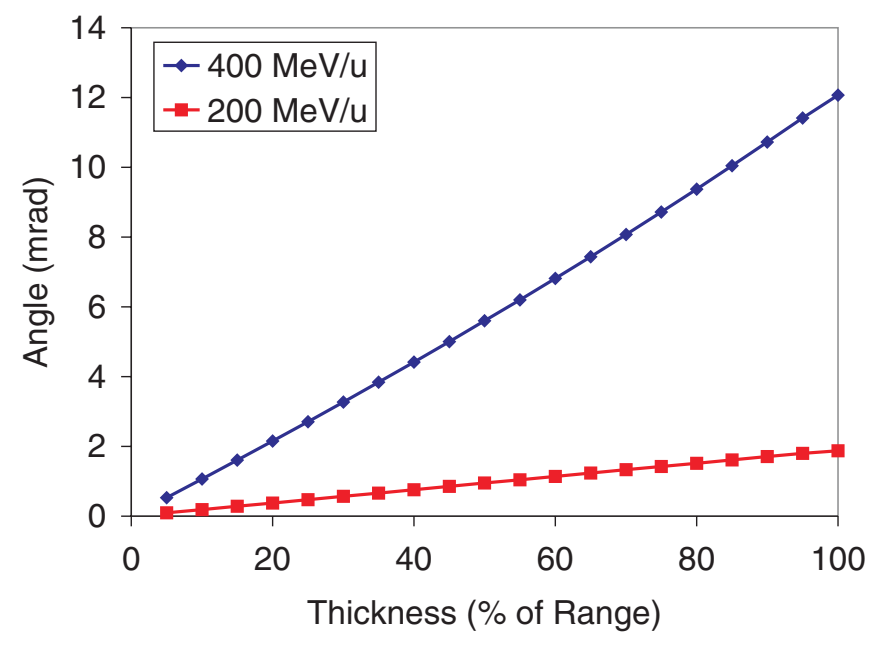

FIG. 5. (Color) Optimal angle needed to maintain linear achromaticity for the case of a ${ }^{132} \mathrm{Sn}$ beam at various wedge thicknesses and two different energies.

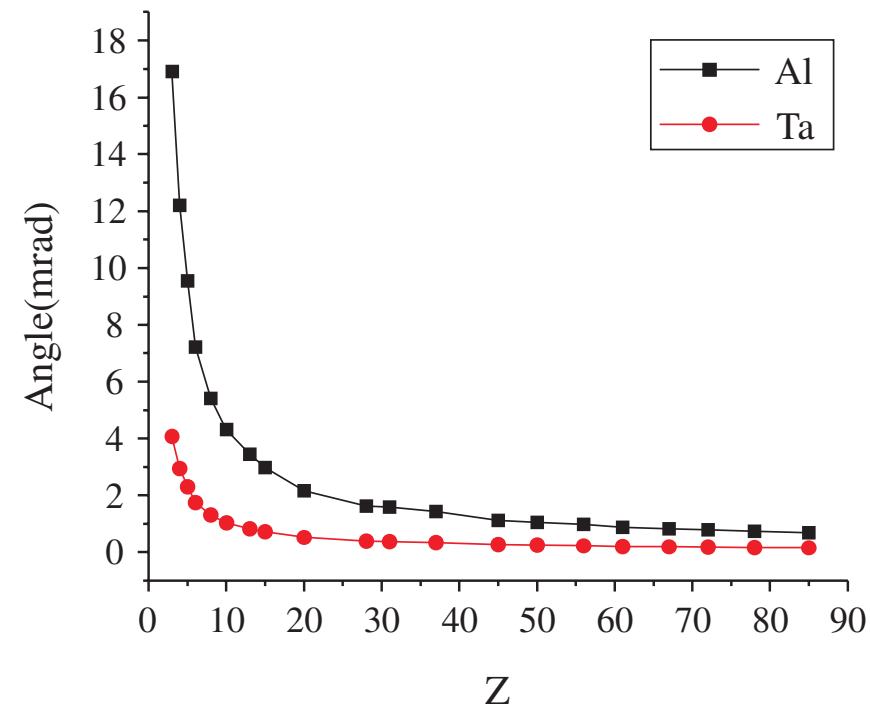

FIG. 6. (Color) Optimal angle needed to maintain linear achromaticity for various beams of $200 \mathrm{MeV} / \mathrm{u}$ energies as a function of $Z$ and two different wedge materials of $30 \%$ range thickness in all cases.

energy of $200 \mathrm{MeV} / \mathrm{u}$ and the thickness of the wedges is the same percentage of the given beam's range, namely, $30 \%$. We can conclude that heavier wedges mean smaller angles and heavier beams also mean smaller angles. However, the right choice of wedge material might be mainly dictated by the atomic and nuclear processes happening in the wedges (the subject of a future publication). As shown in Sec. II, once the optimal angle is found, the main remaining effect of the wedge is the modified magnification. Figure 7 presents the magnification for the case of a ${ }^{132} \mathrm{Sn}$ beam as a function of energy and wedge thickness. Notice that the magnification always increases (it is

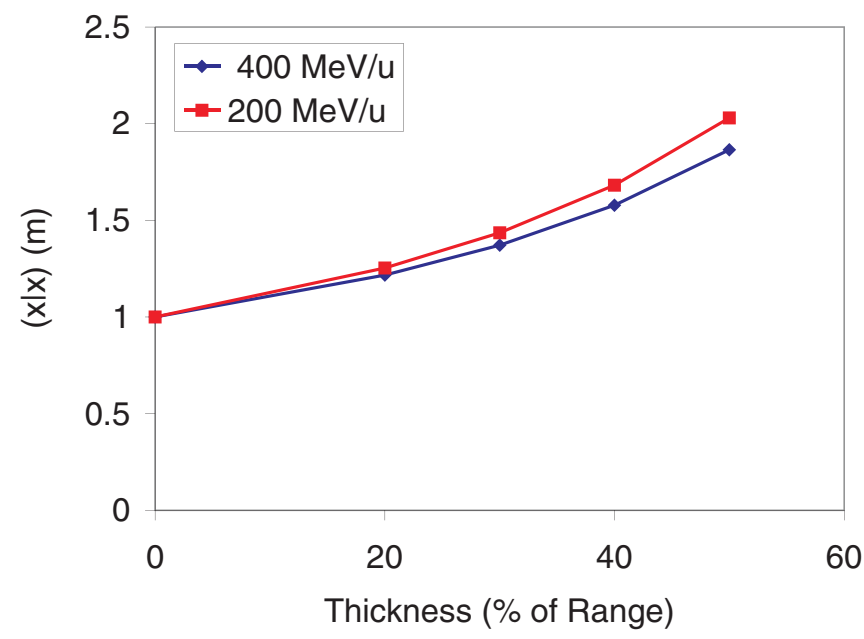

FIG. 7. (Color) The main linear effect of the wedges after shaping them using the optimal angle is the increased magnification. The plot shows the magnification for a ${ }^{132} \mathrm{Sn}$ beam at two different energies as a function of wedge thickness. 
TABLE II. Some map elements that illustrate the effect of the higher order shape of the wedge on the map. The numerical values correspond to a $200 \mathrm{MeV} / \mathrm{u}{ }^{132} \mathrm{Sn}$ beam incident on an aluminum degrader of thickness equal to $30 \%$ of the tin beam range.

\begin{tabular}{lccc}
\hline \hline Case/aberration $[\mathrm{mm}]$ & $(x \mid \delta \delta)$ & $(x \mid a \delta \delta)$ & $(x \mid \delta \delta \delta)$ \\
\hline Wedge angle & 18 & -10 & 2 \\
Wedge angle and curvature & 0 & 1 & 11 \\
Wedge angle, curvature and third order effect & 0 & 1 & 0 \\
\hline \hline
\end{tabular}

unity in absence of the wedge), and grows faster than linearly with thickness, while showing again a very weak energy dependence. For realistic cases the magnification can be as high as 2, which implies a corresponding factor of 2 blowup of the horizontal emittance in a potential second stage of a fragment separator.

Once the linear part of the map, including the wedge angle, is set it becomes interesting to study the effects of higher order shaping of the wedges. In Table II is shown that evaluating the map of the whole system to third order and changing only the second and third order shape coefficients of the wedge some aberrations can be reduced significantly. In other words, the wedge shape may be used to correct aberrations, namely, the nonlinear dispersions that become significant with the introduction of the wedge. Recall that $(x \mid \delta \delta)_{\text {tot }}$ and $(x \mid \delta \delta \delta)_{\text {tot }}$ are zero if the wedge is absent. Also, some other aberrations, such as $(x \mid a \delta \delta)_{\text {tot }}$, that are connected to the nonlinear dispersions are automatically reduced, but not completely canceled. The residuals cannot be corrected by shaping the wedge.

\section{Optimization of the system with energy degraders}

Putting everything together, by designing the high order achromat, shaping the wedge to third order to cancel aberrations, and overall using every possible knob in the system to minimize aberrations according to the methodology outlined in Sec. II, we arrive at a system that is almost free of aberrations to third order, except a few horizontal angle dependent aberrations that are due to different wedge thicknesses seen by particles as a function of incoming angles and the associated additional energy loss. The fitting was done with four multipoles per cell having superimposed quadrupole, sextupole, and octupole components. There are four cells for one stage of the separator. The quadrupoles and sextupoles are in a double mirror-symmetric arrangement (four independent strengths) and the octupoles have a single mirrorsymmetric arrangement (eight independent strengths) [14]. To second order, the envelopes with and without wedges are roughly the same. At third order, due to quite strong octupole strengths needed, the horizontal off-energy envelopes with wedges increase, somewhat reducing the transmission. Unfortunately, we do not have an analytical third order theory that would give insight into the reasons of this; at third order we employ a straightforward numerical optimization. The magnitude and scaling with wedge thickness of the remaining largest aberrations are presented in Fig. 8 for two different energies. The weak energy dependence holds here too. The corresponding shape coefficients needed to minimize aberrations are plotted in

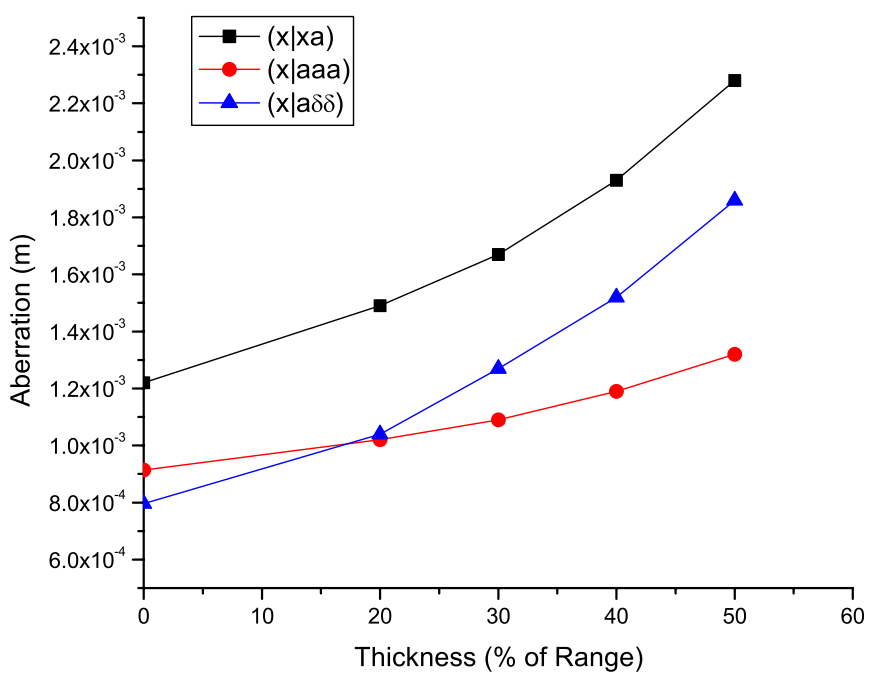

FIG. 8. (Color) The largest remaining aberrations in the system after multipole correction and wedge shaping performed to third order. All aberrations are initial horizontal angle dependent and become larger with increasing wedge thicknesses. The variation with energy is small: $200 \mathrm{MeV} / \mathrm{u}$ case on the left and $400 \mathrm{MeV} / \mathrm{u}$ on the right. 

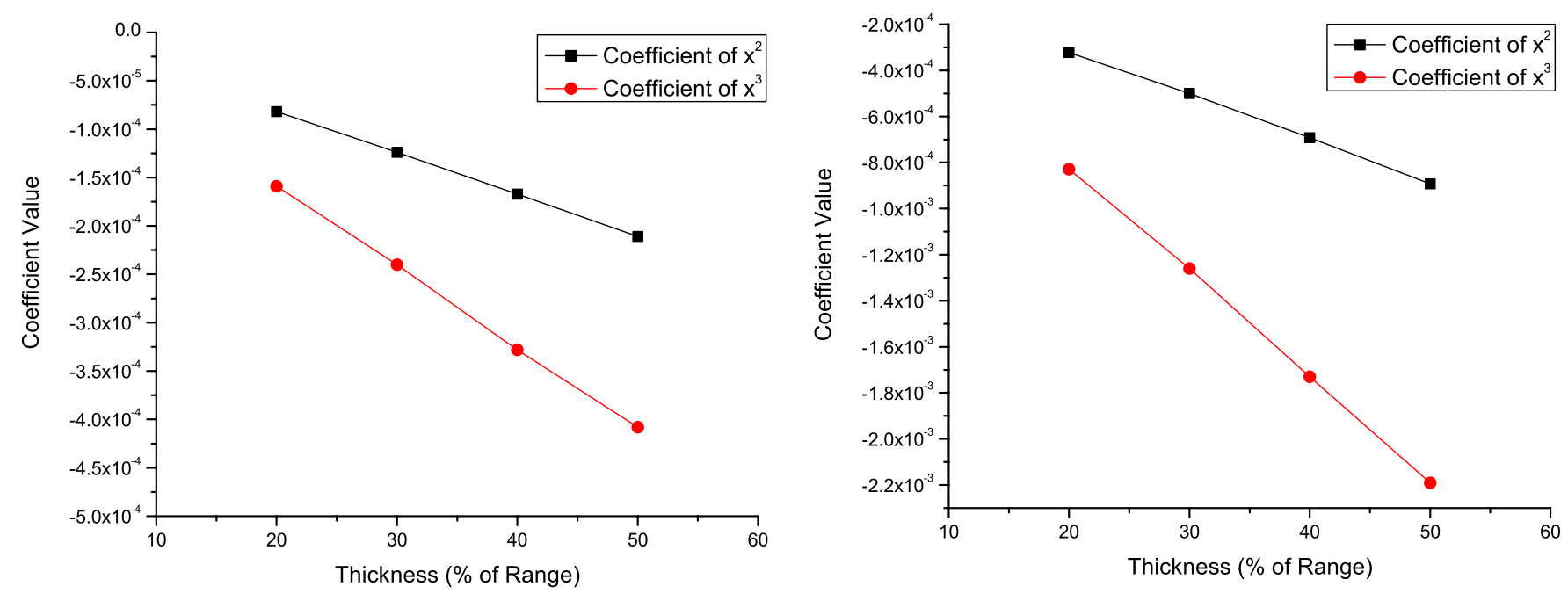

FIG. 9. (Color) Wedge shape coefficients necessary for nonlinear dispersion correction of the cases shown in Fig. 8.

Fig. 9. Notice that at lower energies less curvature is needed. Also, as a function of wedge material the curvature varies with $Z$. Figure 10 presents the case of $\mathrm{Al}$ and $\mathrm{Ta}$ for $200 \mathrm{MeV} / \mathrm{u}$ beams. Although for lighter wedges and particles more curvature is needed, the absolute values are so small as to make the manufacturing of the wedges at these tolerances very difficult, requiring submicron machining precision.

According to the method presented in Sec. II, the aberrations in the full system couple the aberrations of the magnetic optics and those of the wedge. Minimization of aberrations requires simultaneously setting the magnet strengths and shaping the wedge appropriately. The question arises that if one needs to do this kind of optimization for each given rigidity of the beam. Although the magnetic optics scaling with rigidity is well known (this requires one

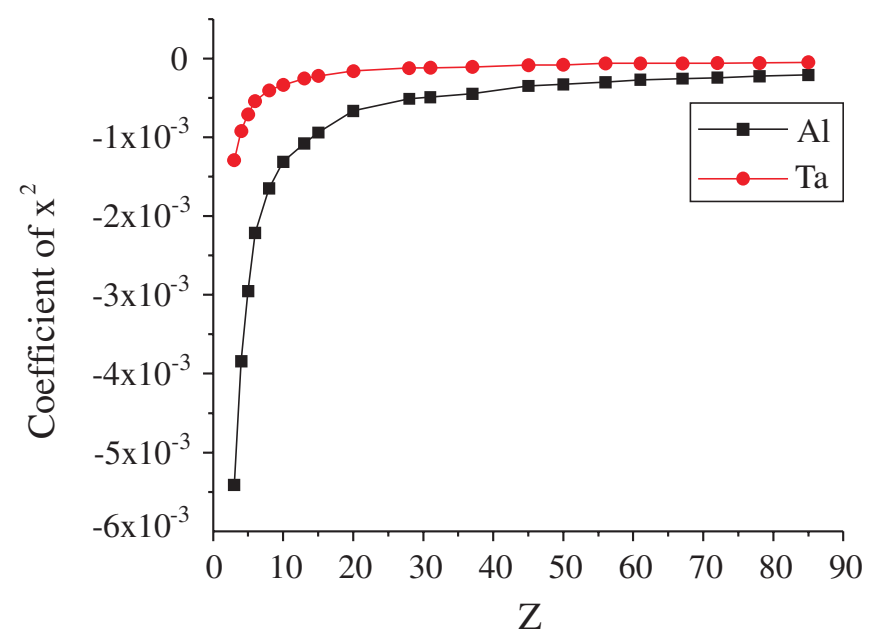

FIG. 10. (Color) Scaling of the wedge curvature for the $200 \mathrm{MeV} / \mathrm{u}$ case as a function of $Z$ for two different wedge materials. The wedge thicknesses are $30 \%$ of the range in each case. to find the magnetic settings for one rigidity, and for all other rigidities these settings are scaled by the new rigidity), it is not obvious that the same scaling still holds true for a system that includes energy degraders. Indeed, we found that no exact scaling holds true in this case. However, fortunately an approximate scaling holds, which means that the setting of sextupoles as a function of rigidity still follows roughly the same scaling law. This is illustrated in Table III, which presents the value of two geometric aberrations at the dispersive image (mainly determined by sextupole settings) needed by the magnetic optics to cancel the same aberrations in the total system at the achromatic image. Note that the approximate scaling holds over a wide range of rigidities. On the other hand, the shaping of the wedge needs to be done on a case-by-case basis. More details can be found in [25].

In all studies up to this point we assumed symmetric wedges in the sense that the entrance and exit shapes are mirror symmetric of each other. Taking into account the difficulty of manufacturing wedges at these tolerances and the prices involved, a practical question arises whether replacing the entrance shape with a face of no angle or higher order coefficients and double the exit face parameters would result in roughly the same map. Indeed, the

TABLE III. Some map elements that illustrate the scaling with rigidity of magnet settings. The numerical values are the coefficients required at the dispersive image for a ${ }^{132} \mathrm{Sn}$ beam incident on an aluminum degrader of thickness equal to $30 \%$ of the tin beam range.

\begin{tabular}{lcc}
\hline \hline Aberration coefficient & \multicolumn{2}{c}{ Value needed @ } \\
\hline & $200 \mathrm{MeV} / \mathrm{u}$ & $1500 \mathrm{MeV} / \mathrm{u}$ \\
\hline$(x \mid a a)$ & -0.9 & -1.9 \\
$(x \mid b b)$ & -0.9 & -1.9 \\
\hline \hline
\end{tabular}


resulting maps differ insignificantly as long as the reference particles see the same thickness. This can the understood by the geometrical arguments of the Appendix.

\section{The resolving power of the fragment separator}

The need for separating isotopes brings up the question of resolution. Since we are interested in particles of certain mass and certain charge, it follows that there are two kinds of resolution: mass resolution for fixed charge state and charge resolution for fixed mass. When both are allowed to vary the best way to visualize what is going on is to plot a cut in the mass and charge dispersion plane along which isotopes are transmitted, hence selected. The resolving powers will depend on the order of the map; at higher order aberrations are included in the image width, decreasing the resolving power. For the estimation of the nonlinear resolving powers realistic initial conditions were used that resulted from the fission of a ${ }^{238} \mathrm{U}$ beam on a liquid lithium target.

For insight, the linear resolving power can be computed analytically from the transfer matrix. Using the notation of the previous sections, it can be shown that the mass and charge resolving powers, respectively, are given by

$$
\begin{aligned}
R_{A, Z} & =\left|\frac{\left(x \mid \delta_{A, Z}\right)_{\mathrm{tot}}}{2 x_{0}(x \mid x)_{\mathrm{tot}}}\right| \\
& =\left|\frac{(x \mid \delta)\left(\delta \mid \delta_{A, Z}\right)_{w}+\left(x \mid \delta_{A, Z}\right)\left(1-(\delta \mid \delta)_{w}\right)}{2 x_{0}(\delta \mid \delta)_{w}}\right|,
\end{aligned}
$$

where $\left(x \mid \delta_{A, Z}\right)_{\text {tot }}$ are the total mass and charge dispersions of the full system, $(x \mid x)_{\text {tot }}$ is the magnification of the full system, $\left(x \mid \delta_{A, Z}\right)$ are the mass and charge dispersions, respectively, of the first half of the fragment separator, and $2 x_{0}$ is the initial horizontal beam size. We implicitly assumed the unit magnification of the magnetic optics,

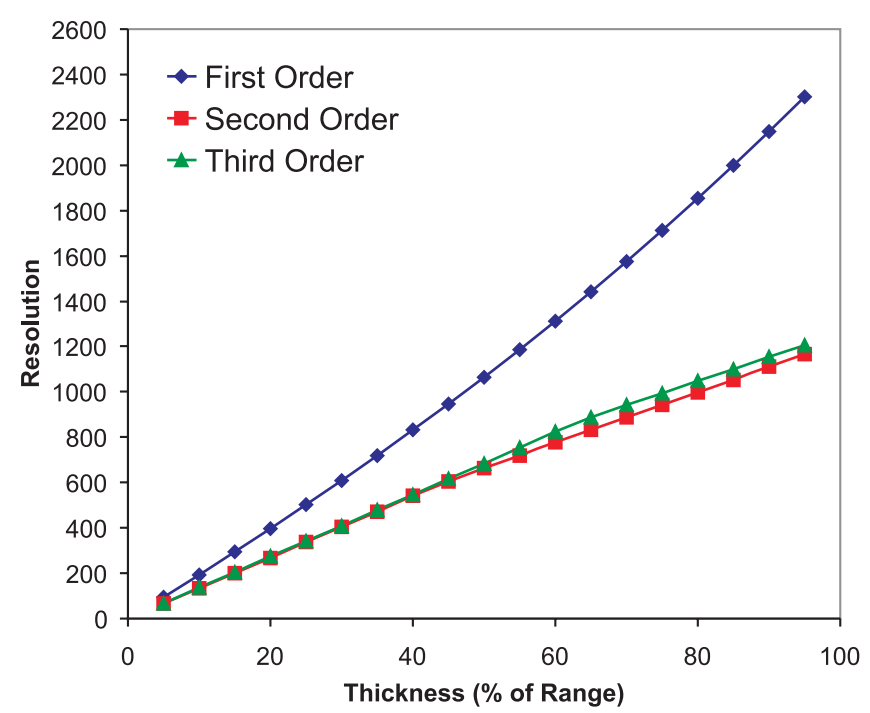

$(x \mid x)=-1$. Maximization of the resolution is equivalent to maximization of (32). However, since $(x \mid \delta)>0$, $\left(x \mid \delta_{A, Z}\right) \lessgtr 0, \quad\left(\delta \mid \delta_{A, Z}\right)_{w}>0$, and $(\delta \mid \delta)_{w}>1$, and all wedge map elements change with the thickness of the wedge, it is not obvious which thickness maximizes resolution. Indeed, it might happen that at certain thicknesses the resolving power vanishes. In practice we noticed this to be the case in certain energy regimes for the charge resolution but not for mass resolution.

Figure 11 shows the resolutions at $400 \mathrm{MeV} / \mathrm{u}$. Aberrations reduce resolutions and tend to switch the slope of the curve from a slope larger than one to a slope slightly smaller than one at larger wedge thicknesses. However, for the determination of the optimal thickness the resolving power computed from the map must be folded with the straggling, which could push the optimal thickness toward smaller values. At lower energy both mass and charge resolving powers increase monotonically with wedge thickness (Fig. 12). Interestingly, the resolving powers are almost invariant with respect to energy. While low energy favors larger mass resolving powers, high energy is better for charge resolution.

The similarity of the resolution data at 200 and $400 \mathrm{MeV} / \mathrm{u}$ suggest that it would be interesting to look at the linear resolutions in a wider energy range to capture the systematic variations of the resolving powers as a function of energy. Interestingly, for three different wedge thicknesses, Fig. 13 shows that around $300 \mathrm{MeV} / \mathrm{u}$ the charge resolving powers vanish. An intuitive explanation of the minimum resolution relies on the nature of the energy-loss dependence on particle parameters. In the absence of the wedge the system is achromatic and the resolution is zero. That means that any two particles with different rigidities due to different charge states and same initial position will end up at the same final position at the

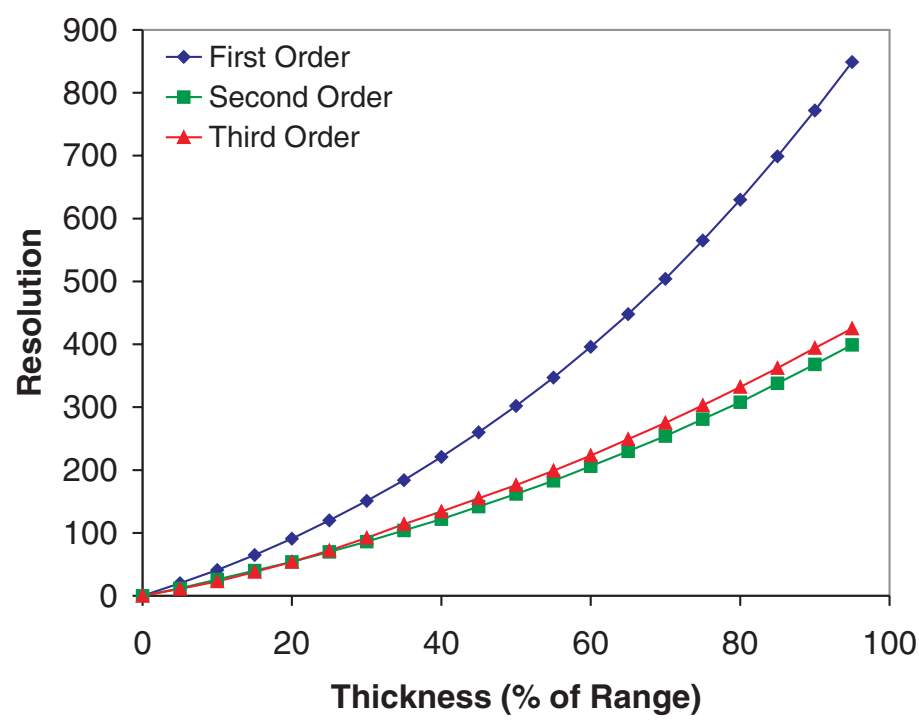

FIG. 11. (Color) Mass (left) and charge (right) resolving powers for a $400 \mathrm{MeV} / \mathrm{u}{ }^{132} \mathrm{Sn}$ beam and Al wedge as a function of wedge thickness evaluated at different map orders. 

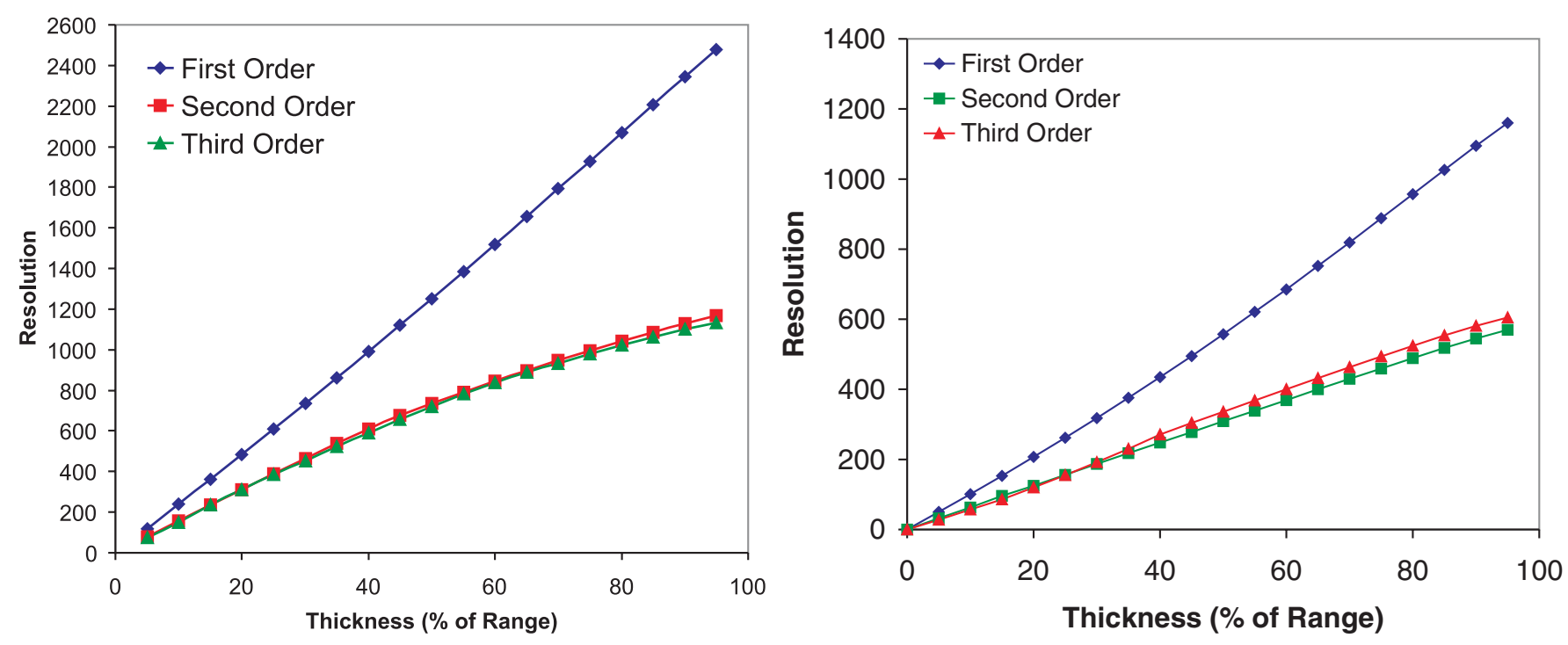

FIG. 12. (Color) Mass (left) and charge (right) resolving powers for a $200 \mathrm{MeV} / \mathrm{u}^{132} \mathrm{Sn}$ beam and Al wedge as a function of wedge thickness evaluated at different map orders.
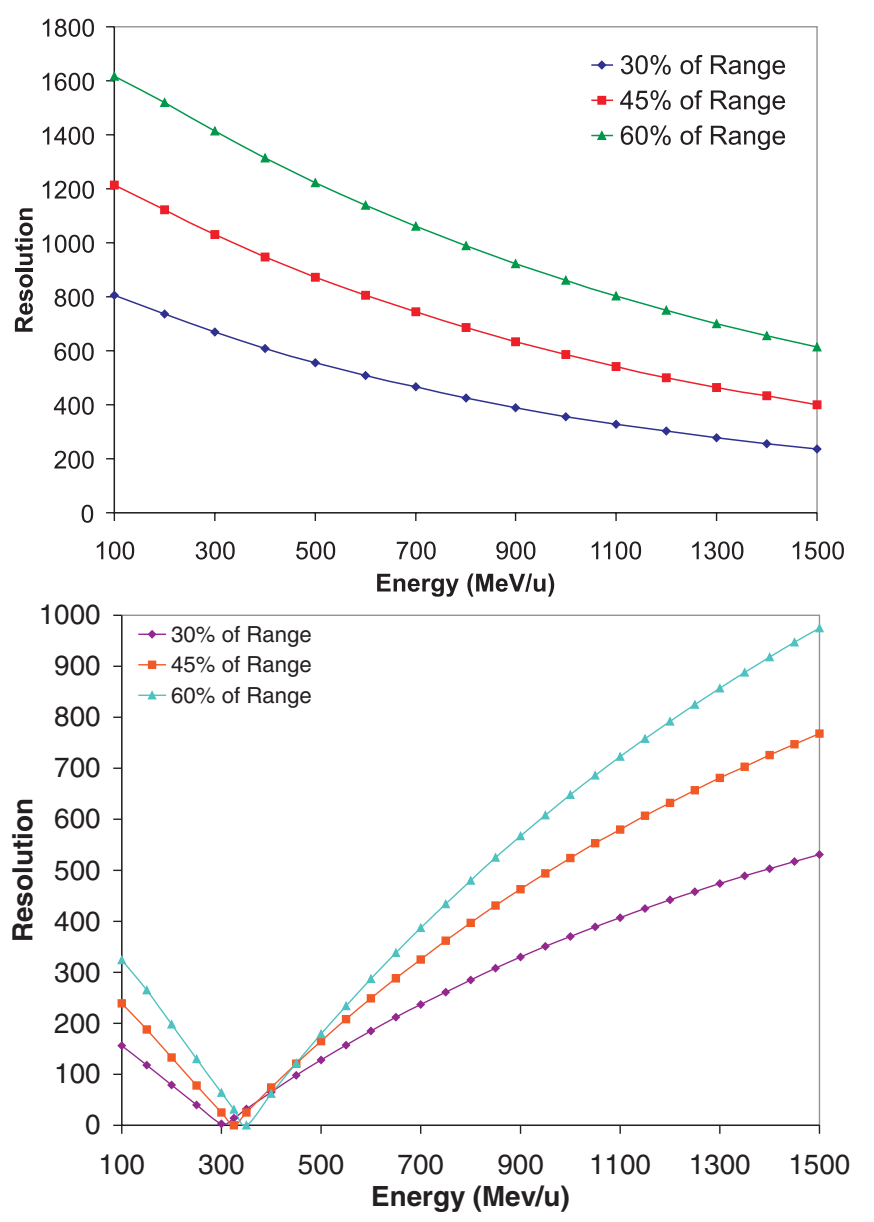

FIG. 13. (Color) Mass (upper) and charge (lower) linear resolving powers as a function of energy for three different wedge thicknesses. achromatic image point. With the introduction of the wedge into this achromatic system (such that the new system maintains achromaticity), the relative rigidity dispersion of the particles will change in general after traversing the wedge, introducing a net dispersion at the end of the system. However, for certain energies and wedge thicknesses two competing effects cancel each other: on one hand, the higher charge states are less rigid, so they bend more and will see smaller thicknesses of the wedge than the lower charge states. On the other hand, the charge dependence of the energy loss implies that the higher charge state has a larger energy-loss rate, effectively canceling the fact that the particle goes through a thinner portion of the wedge. The net effect is that in some cases the particles will not change their relative rigidities leaving the wedge, that in turn will not induce a net charge dispersion at the end of the system. In other words, in these cases the wedge becomes transparent to the system. Figure 13 shows when this happens. Mathematically, the terms due to these two competing effects are obvious from (32).

If both mass and charge are allowed to vary, the separation cut shape and orientation becomes worthwhile to study. In the linear approximation the cut is obviously a line in the $\left(\delta_{A}, \delta_{Z}\right)$ plane, and the slope is given by

$$
\frac{\left(\delta \mid \delta_{A}\right)_{w}+\left(x \mid \delta_{A}\right)(\delta \mid x)_{w}}{\left(\delta \mid \delta_{Z}\right)_{w}+\left(x \mid \delta_{Z}\right)(\delta \mid x)_{w}} .
$$

Interestingly, if nonlinearities are taken into account, at least visually, the separation cut stays roughly a line. Figure 14 presents the separation cut evaluated up to third order map elements for the case of a $400 \mathrm{MeV} / \mathrm{u}{ }^{132} \mathrm{Sn}$ beam and a $45 \%$ thickness aluminum wedge. Therefore, one can look at the separation cut angle in the $\left(\delta_{A}, \delta_{Z}\right)$ 


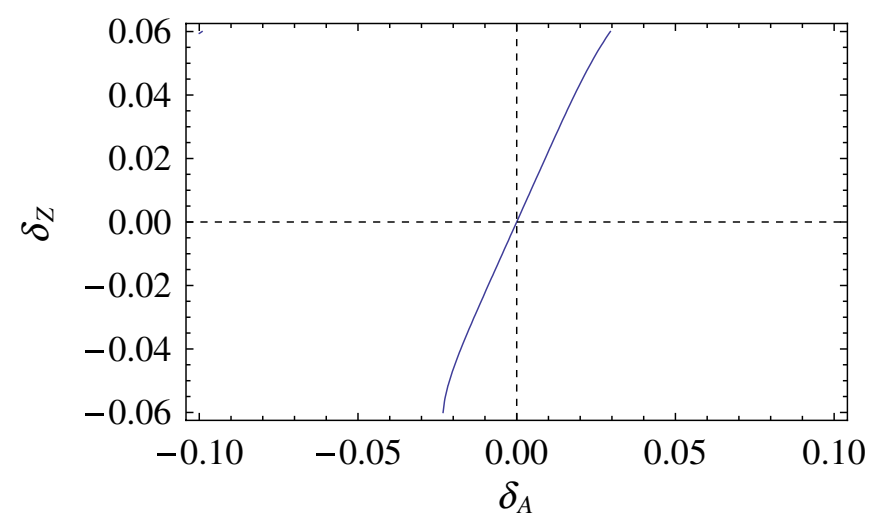

FIG. 14. (Color) Separation cut including nonlinearities up to third order. The origin corresponds to a $400 \mathrm{MeV} / \mathrm{u}{ }^{132} \mathrm{Sn}$ beam and wedge thickness corresponding to $45 \%$ of the range.

plane to illustrate the effect of the wedges. As before for other quantities, the slope will depend on wedge thickness and beam energy. Figure 15 shows several energy cases at a variety of wedge thicknesses and wedge materials. It is clear that, while at higher energies changing the wedge thickness affects significantly the slope, at lower energies the separation cut angle is almost constant for a wide range of thicknesses. While the material of the wedge does influence the cut angle a little bit, at lower energies this influence is not sufficient. This result unfortunately implies that some of the benefits of a second stage of separation diminish at lower energies. Nonetheless, the total mass resolving powers of two-stage separators will increase with respect to single-stage separators given the right bending orientation, which will improve overall the separation purity.

Finally, to clarify the relationships among the resolving powers and separation cut lines, note that in reality the separation cut is not exactly a zero thickness line. Because of the finite width of the selection slit particles having slightly off-centered final $x$ coordinates will be also selected along separation cut lines with the same slope, but slightly off centered as well. Therefore, in reality the selection cuts consisting of the many lines form a separation cut band. It is easy to see that, if the slit width is set according to the image width of the beam to be selected, then the thickness of the separation cut band along the mass and charge coordinate axes are inversely proportional to the mass and charge resolving powers, respectively. Hence, high resolving powers benefit the separation purity even for two-stage separators where the separation cut bands can be rotated. Of course, it is even more important for one-stage separation. Finally, vanishing mass and charge resolving powers are equivalent to separation cut angles equal to $0^{\circ}$ and $90^{\circ}$, respectively.

\section{E. Summary and conclusions}

Next-generation fragment separators will be crucial for the success of future exotic beam facilities. These fragment separators, besides the sophisticated magnetic optics, need precise energy degraders in order to be able to deliver the performance required of these instruments.

We developed a second order analytic and an arbitrary order numerical procedure for the computation of wedge maps. These maps may be included with the rest of the system and, through composition, the map of the full system can be computed, analyzed, and optimized. The numerical procedure was implemented in an extended version of the code COSY INFINITY. The analytic theory gives insight into the design optimization of an achromatic stage of a separator.

We derived the conditions necessary for maintaining linear achromaticity. The optimal angle (thickness gradient in the dispersive plane) can be easily fit, and we illustrated its variation as a function of a variety of parameters. We concluded that the main linear residual effect of the wedge is an increased magnification and corresponding loss of resolving power, and the associated increase in beam emittance. We also proved that by introducing just the right amount of wedge curvature and higher order effects all
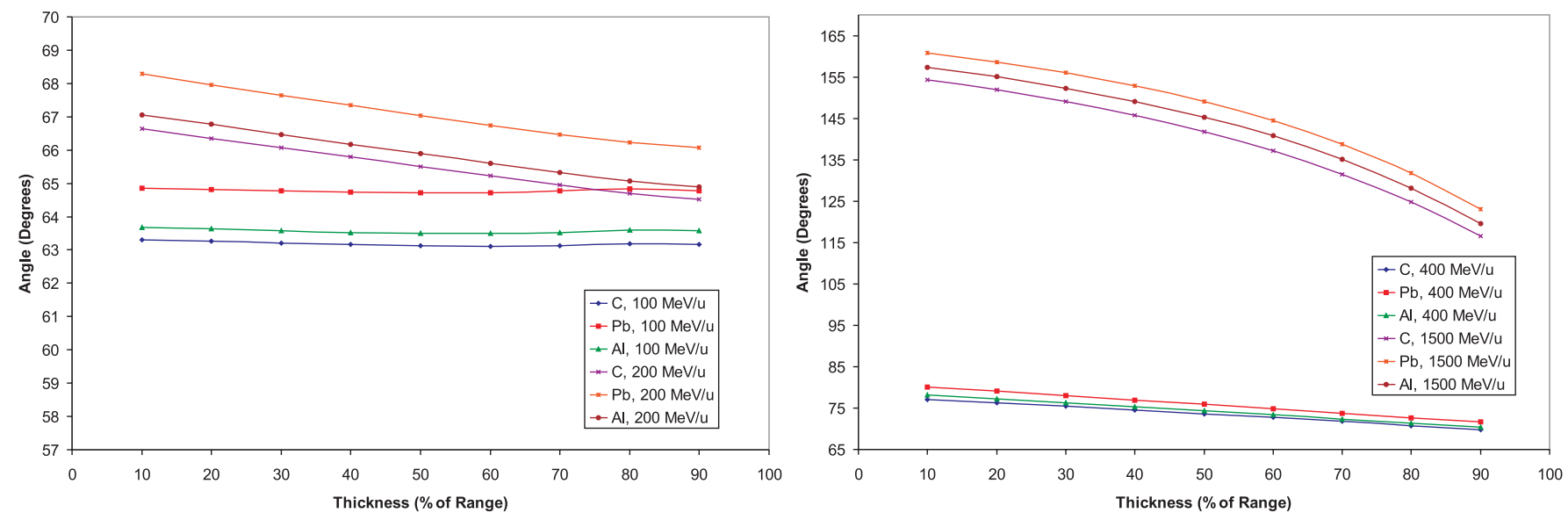

FIG. 15. (Color) Separation cut angles at different energies of a ${ }^{132} \mathrm{Sn}$ beam as a function of wedge material and thickness. 
second order and most third order aberrations can be canceled for a pointlike beam. Unfortunately, the required machining precision for practical cases might be difficult to achieve. Also, even for a perfect shaping some residual aberrations are unavoidable, mainly due to particles' horizontal angle dependence of the effective thickness. We noticed that the third order off-energy envelopes are larger with wedge due to quite strong octupoles needed. We studied the scaling of the remaining aberrations as a function of wedge thickness. We also found that an approximate scaling of the magnetic field strengths with rigidity still holds for systems including energy degraders, but the shaping of the wedges needs to be done on a case-by-case basis.

Studies of mass and charge resolving powers and separation cuts showed interesting behavior. Under certain conditions the charge resolving power might vanish. In general, thicker wedges maximize resolution (in the absence of stochastic effects). At fixed energy the mass resolving powers are monotonic functions of wedge thickness. This is not true for charge resolving power; there is an energy range around $300 \mathrm{MeV} / \mathrm{u}$ that exhibits more complicated behavior. Also, the separation cuts are lines even in the presence of nonlinearities and we studied the associated slope in the mass-charge dispersion plane. We concluded that the cut angle varies significantly as a function of thickness at higher energies and it flattens out at lower energies. Therefore, two-stage separators are more useful at higher energies.

Further work will involve studies of two-stage fragment separators and fragment separators for gas cells. For studies of separation purity and background distribution, the techniques developed in this paper will be combined with Monte Carlo techniques for a hybrid map-Monte-Carlo extension of COSY INFINITY.

\section{ACKNOWLEDGMENTS}

We would like to thank Martin Berz and his group for assistance with wedge map-related COSY work. This work was supported by the U.S. Department of Energy, Office of Nuclear Physics, under Contract No. DE-AC02$06 \mathrm{CH} 11357$.

\section{APPENDIX A: PROOF OF EQ. (10)}

Since this is a linear calculation, it is sufficient to consider a wedge of thickness $l_{w}$ and half-angle $\phi$ with no curvature or higher order shape effects. The situation is depicted in Fig. 16. Consider two particles of the same energy in the midplane, one with $x_{i}=0, a_{i} \neq 0$ and another one with $x_{i} \neq 0, a_{i}=0$. The particles traverse the wedge and see effective thicknesses equal to their track lengths $l_{a}$ and $l_{x}$, respectively. At the end, the particles will emerge with new relative energy deviations given by $\delta_{a, f}=(\delta \mid a)_{w} a_{i}$ and $\delta_{x, f}=(\delta \mid x)_{w} x_{i}$, respectively. If it

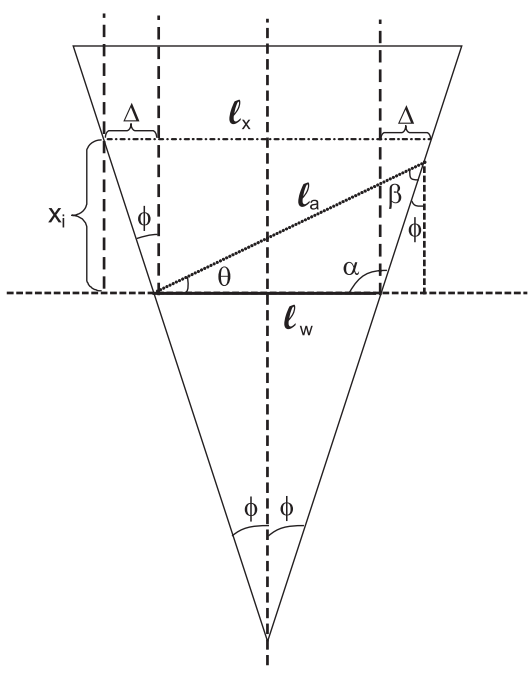

FIG. 16. Geometric schematic of the wedge that illustrates the quantities needed for the proof of (10).

happens that the track lengths are equal $l_{x}=l_{a}$ then also the energies must be the same, giving $\delta_{x, f}=\delta_{a, f}$. The calculation of the track lengths is a geometric problem that can be easily solved with the help of Fig. 16.

To calculate $l_{x}$ notice that it is equal to $l_{w}+2 \Delta$, where $\Delta=x_{i} \tan \phi$. Hence, $l_{x}=l_{w}+2 x_{i} \tan \phi$. Calculation of $l_{a}$ is just a little bit more involved. Applying the sin theorem we obtain

$$
\frac{\sin \beta}{l_{w}}=\frac{\sin \alpha}{l_{a}},
$$

and the following relationships among the various angles: $\theta+\alpha+\beta=\pi$ and $(\pi-\alpha)+\pi / 2+\phi=\pi$. It is well known that to first order $a_{i}=\theta$. It follows that

$$
l_{a}=l_{w} \frac{\cos (\phi)}{\cos (\theta+\phi)},
$$

which reduces at first order to $l_{a}=l_{w}(1+\theta \tan \phi)=$ $l_{w}\left(1+a_{i} \tan \phi\right)$. Therefore, $l_{x}=l_{a}$ for given wedge parameters if $x_{i} / a_{i}=l_{w} / 2$, independent of the wedge angle. In this case the equal energies $\delta_{x, f}=\delta_{a, f}$ result in the relation

$$
(\delta \mid x)_{w} \frac{l_{w}}{2}=(\delta \mid a)_{w},
$$

which is (10), and the proof is complete. Clearly, extending the geometrical arguments to higher order similar equations can be derived that connect higher order wedge map elements.

[1] Opportunities in Nuclear Science, edited by J. Symons, technical report, NSAC, 2002.

[2] RISAC, Scientific Opportunities with a Rare-Isotope Facility in the United States, technical report, NRC, 2006. 
[3] National Research Council of the National Academies Rare-Isotope Science Assessment Committee, Scientific Opportunities with a Rare-Isotope Facility in the United States, technical report, NAS, 2007.

[4] Y. Yano, in Proceedings of the 17th International Conference on Cyclotrons and their Applications, Tokyo, Japan, 2004, pp. 169-173.

[5] W. Henning, Nucl. Phys. A734, 654 (2004).

[6] J. Nolen, Nucl. Phys. A734, 661 (2004).

[7] B. M. Sherrill, Nucl. Instrum. Methods Phys. Res., Sect. B 204, 765 (2003).

[8] J.P. Dufour and R. Del Moral, Nucl. Instrum. Methods Phys. Res., Sect. A 248, 267 (1986).

[9] H. Geissel et al., Nucl. Instrum. Methods Phys. Res., Sect. B 70, 286 (1992).

[10] H. Geissel, H. Weick, M. Winkler, G. Munzenberg, and M. Yavor, Nucl. Instrum. Methods Phys. Res., Sect. B 247, 368 (2006).

[11] D. J. Morrissey, B. M. Sherrill, M. Steiner, and I. Wiedenhoever, Nucl. Instrum. Methods Phys. Res., Sect. B 204, 90 (2003).

[12] T. Kubo, Nucl. Instrum. Methods Phys. Res., Sect. B 204, 97 (2003).

[13] H. Geissel, H. Weick, M. Winkler, G. Munzenberg, and M. Yavor, Nucl. Instrum. Methods Phys. Res., Sect. B 204, 71 (2003).

[14] B. Erdelyi, J. Maloney, and J. A. Nolen, Phys. Rev. ST Accel. Beams 10, 064002 (2007).
[15] K.-H. Schmidt, E. Hanelt, H. Geissel, G. Munzenberg, and J. P. Dufour, Nucl. Instrum. Methods Phys. Res., Sect. A 260, 287 (1987).

[16] M. Berz, Modern Map Methods in Particle Beam Physics (Academic Press, San Diego, 1999).

[17] B. Erdelyi, L. Bandura, J. Nolen, and S. Manikonda, in Proceedings of the 2007 Particle Accelerator Conference, Albuquerque, New Mexico, 2007 (IEEE, Albuquerque, New Mexico, 2007), p. 3576.

[18] O. B. Tarasov and D. Bazin, Nucl. Instrum. Methods Phys. Res., Sect. B 266, 4657 (2008).

[19] M. Mazzocco, D. Ackermann, M. Block, H. Geissel, F. Hertfurth, F. P. Hesberger, S. Hofmann, N. Iwasa, K. Nishio, W. R. Plas, C. Scheidenberger, H. Weick, M. Winkler, and The SHIPTRAP Collaboration, Nucl. Instrum. Methods Phys. Res., Sect. B 266, 3467 (2008).

[20] B. Erdelyi (to be published).

[21] S. Wolfram, The MATHEMATICA Book (Wolfram Media, Champaign, IL, 2003), 5th ed.

[22] H. Weick (private communication).

[23] J. Grote, M. Berz, and K. Makino, Nucl. Instrum. Methods Phys. Res., Sect. A 558, 106 (2006).

[24] K. Makino and M. Berz, Nucl. Instrum. Methods Phys. Res., Sect. A 558, 346 (2006).

[25] L. Bandura, B. Erdelyi, and J. Nolen, in Proceedings of the 2007 Particle Accelerator Conference, Albuquerque, New Mexico, 2007, Ref. [17], p. 3426. 